\title{
Distance Constrained Mapping to Support NoC Platforms Based on Source Routing ${ }^{\star}$
}

\author{
Rafael Tornero ${ }^{1}$, Shashi Kumar ${ }^{2}$, Saad Mubeen ${ }^{2}$, and Juan Manuel Orduña ${ }^{1}$ \\ 1 Departamento de Informática, Universitat de València, Spain \\ \{Rafael.Tornero, Juan.Orduna\}@uv.es \\ 2 School of Engineering, Jönköping University, Sweden \\ \{Shashi.Kumar, mems07musa\}@jth.hj.se
}

\begin{abstract}
Efficient $\mathrm{NoC}$ is crucial for communication among processing elements in a highly parallel processing systems on chip. Mapping cores to slots in a NoC platform and designing efficient routing algorithms are two key problems in NoC design. Source routing offers major advantages over distributed routing especially for regular topology NoC platforms. But it suffers from a serious drawback of overhead since it requires whole communication path to be stored in every packet header. In this paper, we present a core mapping technique which helps to achieve a mapping with the constraint over the path length. We have found that the path length constraint of just $50 \%$ is sufficient in most cases. We also present a method to efficiently compute paths for source routing leading to good traffic distribution. Evaluation results show that performance degradation due to path length constraint is negligible at low as well as high communication traffic.
\end{abstract}

Keywords: Network on Chip, Core Mapping, Routing Algorithms, Source Routing.

\section{Introduction}

As Semi-conductor Technology advances, it becomes possible to integrate a large number of Intellectual Property (IP) cores, like DSPs, CPUs, Memories, etc, on a single chip to make products with complex and powerful functions. Efficient communication infrastructure is crucial for harnessing enormous computing power available on these Systems on Chip (SoCs). Network on Chip (NoC) is being considered as the most suitable candidate for this job [1].

Many design choices and aspects need to be considered for designing a SoC using NoC paradigm. These include: network topology selection, routing strategy selection and application mapping. Both application mapping and routing strategy have big impact on the performance of the application running on a NoC platform. The application mapping problem consist of three tasks: i) the

\footnotetext{
* This work has been jointly supported by the Spanish MEC and European Commission FEDER funds and the University of Valencia under grants Consolider Ingenio2010 CSD2006-00046 and TIN2009-14475-C04-04 and V-SEGLES-PIE Program.
} 
application is split into a set of communication tasks, generally represented as a task graph; ii) the tasks are assigned and scheduled on a set of IP cores selected from a library; iii) the IP cores have to be placed onto the network topology in such a way that the desired metrics of interest are optimized. The first two steps are not new since they have been extensively addressed in the area of hardware/software co-design and IP reuse 2] by the CAD community. The third step, called topological mapping, has recently been addressed by a few research groups [3], 4].

One way to classify the routing algorithms is by considering the component in the network where the routing decision to select the path is done. Under this consideration the routing algorithms are classified into source routing and distributed routing algorithms. In source routing algorithms, the path between each pair of communicating nodes is computed offline and stored at each source node. When a core needs to communicate with another core the encoded path information is put in the header of each packet. In distributed routing, the header only needs to carry the destination address and each router in the network has competence to make the routing decision based on the destination address.

Source routing has not been considered so far for NoCs due to its perceived underutilization of network bandwidth due to the requirement of large number of bits in the packet header to store path information. This conclusion may be valid perhaps for large dynamic networks where network size and topology are changing. But in a NoC with fixed and regular topology like mesh, the path information can be efficiently encoded with small number of bits. Saad et. al. [5] have made a good case for use of source routing for mesh topology NoCs. It can be easily shown [6] that two bits are sufficient to encode information about one hop in the path. Since the packet entering a router contains the pre-computed decision about the output port, the router design is significantly simplified. Also, since NoCs used in embedded systems are expected to be application specific, we can have a good profile of the communication traffic in the network [7. This allows us to offline analyze the traffic and compute efficient paths according to the desired performance characteristics, like uniform traffic load distribution, reserved paths for guaranteed throughput etc.

Figure 1 shows an application, that has been assigned and scheduled on eight cores, topologically mapped on a $4 \times 2$ mesh. The Application Characterization Graph (APCG) of this application can be seen in Figure 1(a), where a node corresponds to a core and a directed edge corresponds to communication between two connected cores. APCG will be defined more formally in section 2, Assuming minimal routing, the maximum route length required is equal to the diameter of the topology. It means that, if the diameter was used for this example, the required path length would be 4 hops and therefore 10 bits would be required to code a route (see Figure 1(b)). However, it is possible to find a mapping in which the maximum distance between two communicating cores is much smaller than the diameter. Figure 1(c) shows such a mapping for the example in which the maximum distance is just two hops and only 6 bits are required for the path information. 


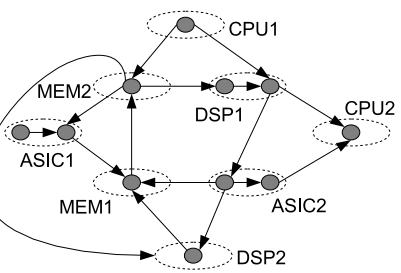

(a)

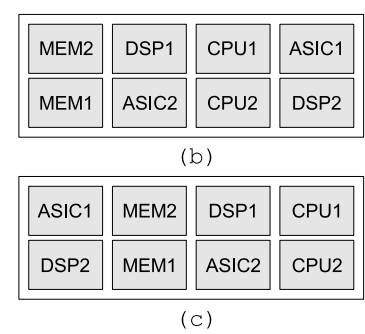

(c)

Fig. 1. Different mappings of the same application. a) the APCG, b) A distance unconstrained mapping, c) A 2-hops constrained mapping.

Close compactness of mapping could lead to higher congestion in certain links. Since routers for source routing are relatively faster than routers for distributed routing, the above disadvantage will be adequately compensated [6].

\section{Related Work}

A large number of routing algorithms have been proposed in literature for NoCs. Most proposals fall in the category of distributed adaptive routing algorithms and provide partial adaptivity thus providing more than one path for most communicating pairs and at the same time avoiding possibility of deadlocks. In [7], Palesi et al. propose a methodology to compute deadlock free routing algorithms for application specific NoCs with the goal of maximizing the communication adaptivity.

Several works have been proposed in literature in the context of core mapping. $\mathrm{Hu}$ et al. present a branch and bound algorithm for mapping cores in a mesh topology NoC architecture that minimizes the total amount of power consumed in communications $[8$. Murali et al. present a work to solve the mapping problem under bandwidth constraint with the aim of minimizing the communication delay by exploiting the possibility of splitting the traffic among various paths [3. Hansson et al. present an unified single objective algorithm which couples path selection, mapping of cores, and channel time-slot allocation to minimize the network required to meet the constraints of the application [9]. Tornero et al. present a communication-aware topological mapping technique that, based on the experimental correlation of the network model with the actual network performance, avoids the need to experimentally evaluate each mapping explored [4. In [10, Tornero et al. present a multi-objective strategy for concurrent mapping and routing for NoC. All the aforementioned works address the integration of mapping and routing concurrently but taking into account only the distributed routing functions.

Although source routing has been shown to be efficient for general networks 11, it had not been explored so far for NoC architectures. Recently Mubeen et.al [5] have made a strong case for source routing for small size mesh topology NoCs. The author, has demonstrated that source routing can have higher communication performance than adaptive distributed routing [6]. In this paper 
we modify our earlier approach and tackle the integration of topological mapping for NoC platforms which use limited path length source routing for inter-core communication.

\section{Problem Formulation}

Simply stated, our goal is to find an arrangement of cores in tiles together with path selection such that the global communication cost is minimized and the maximum distance among communicating cores is within the given threshold. The value of the threshold comes from the fixed on-chip communication infrastructure (NoC) platform which uses source routing with an upper limit on the length of the path. Before formally defining the problem, we need to introduce the following definitions [8].

Definition 1. An Application Characterization Graph $A P C G=G(C, A)$ is a directed graph, where each vertex $c_{i} \in C$ represents a selected IP core, and each directed arc $a \in A$ characterizes the communication process from core $c_{i}$ to core $c_{j}$. For each communication $a \in A \wedge a=\left(c_{i}, c_{j}\right)$, the function $B(a)$ returns the bandwith requirements of $a$. This is minimum bandwith that should be allocated by the network in order to meet the performance constraint for communication $a$.

Definition 2. An Architecture Characterization Graph $A R C G=G(T, L)$ is a directed graph which models the network topology. Each vertex $t_{i}$ represents a tile, and each directed arc $l_{i j}$ represents the channel from tile $t_{i}$ to tile $t_{j}$.

We must solve two problems: first, we have to find a mapping within the constraint of maximum distance allowed by the communication platform. The second problem to be solved is to compute efficient paths for all communicating pairs of cores such that there is no possibility of deadlock as well as the traffic is well balanced. We can formulate the first problem as follows. Given the APCG and the ARCG, that satisfy $|C| \leqslant|T|$, find a mapping function $M$ from $C$ to $T$ which minimizes the mapping cost function $M_{c}$ :

$$
\min \left\{M_{c}=\sum_{\substack{\forall c_{i}, c_{j} \in C \\ a=\left(c_{i}, c_{j}\right) \in A}} B(a) *\left(\operatorname{dist}\left(M\left(c_{i}\right), M\left(c_{j}\right)\right)\right)^{3}\right\}
$$

such that:

$$
\operatorname{dist}\left(M\left(c_{i}\right), M\left(c_{j}\right)\right) \leq \text { Threshold } \wedge a=\left(c_{i}, c_{j}\right) \in A
$$

In the equation 1, the second term of the summation is raised to power 3 with the aim of giving more importance to the distance in the search for a pseudo-optimal mapping. This value is a trade-off between the quality of the results and the computation time (the power 2 provides poor quality of results and power of 4 and higher ones are too much time consuming). Condition 2 
guarantees that every pair of communicating cores should be mapped such that the Manhattan distance between them is less than the threshold. We assume that the underlying source routing uses only minimal distances. Nevertheless, this threshold cannot be smaller than the lower bound on the path length required for mapping APCG on ARCG. The lower bound in this context refers to a value such that any possible mapping will have at least one pair with distance more than or equal to the lower bound. For example, the lower bound for the APCG in our example is 2, since there is no possibility to find a mapping with distance 1. The APCG together with the ARCG can be analyzed in order to find the lower bound for the mapping.

In a $4 \times 2$ mesh topology, a node can be connected to maximum three other cores with a distance 1 . A core can be connected to up to 6 cores if distance of 2 hops is allowed. There may not be any 2 distance constrained mapping available for an APCG with maximum out-degree 5. If $L$ is the lower bound, then one can start by searching for a mapping with constraint equal to $L$. If one fails then one must repeat the process of finding a feasible mapping by using $L+1, L+2, \ldots$. and so on as the constraint.

Once the cores are mapped satisfying (2), the second problem is to find a path for every communicating core pair $C_{i}$ and $C_{j}$ such that: the path length is equal to Manhattan distance between $C_{i}$ and $C_{j}$; there is no possibility of deadlock when some or all other core pairs communicate concurrently and the traffic load on all the links in the network is as balanced as possible.

\section{The Distance Constrained Mapping Algorithm}

We have modified our earlier mapping approach developed for NoC platforms using distributed routing techniques [4] to obtain distance constrained topological mapping. This approach considers the network resources and the communication pattern generated by the tasks assigned to different cores in order to map such cores to the network nodes. The method is based on three main steps.

Step 1. Model the network as a table of distances (or costs) between each pair of source/destination nodes. The cost for communicating each pair of nodes is computed as inversely related to the available network bandwidth.

Step 2. Perform a heuristic search in the solution space with the aim of obtaining a near-optimal mapping that satisfies our distance restrictions.

Step 3. Repair the mapping found in the second step if some communicating pairs violate the distance constraints.

We have computed steps 1 and 2 as in our previous work ([4]). If the mapping found by the heuristic method does not satisfy the distance constraint, then a heuristic repair procedure, step 3, tries to repair the solution found. This procedure, based on [12], consists of a hill-climbing that minimizes the number of constraints violated by the solution mapping. The advantage of this procedure 


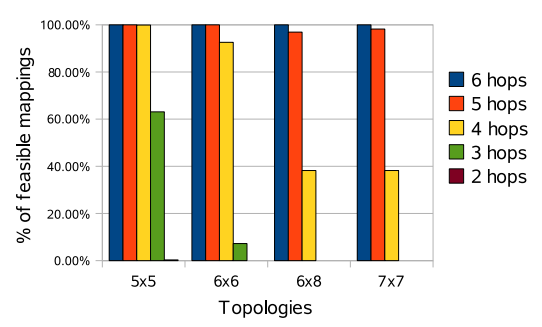

Fig. 2. Feasible mappings with distant constraint

is that it is fast to compute, but presents some drawbacks like the capacity to fall in a local minimum that does not satisfy the distance constraint. This drawback could be reduced by repeating the same $\dddot{i} \iota \frac{1}{2}$ procedure several times.

It should be noticed that the goal of this work is to prove that it is possible to obtain efficient solutions for the problem of distance constrained mapping by using a mapping technique analogous to the one shown in our previous work (4]). In order to achieve this goal, we have used the same heuristic method for solving step 2 than the one shown in 4]. Since that method was not designed for this problem, a new heuristic method specifically developed for solving this problem is likely to provide better solutions.

\subsection{Feasibility Experiments}

In order to test the distance constrained topological mapping method we have made a set of feasibility experiments. These consists of mapping 500 random APCGs on several 2-D mesh topologies. We have used a $5 x 5,6 x 6,6 x 8$ and $7 x 7$ mesh topologies. Each node of each APCG presents an out-degree log-normally distributed with a mean of 2 and a standard deviation of 1 communications. This distribution was motivated by analysis of multi-media applications used in literature 78 . We have used an uniform probability distribution for spatial communications. It means that the probability of a core $c_{i}$ communicating with a core $c_{j}$ is the same for every core. The communication bandwith between each pair of communicating cores is distributed uniformly between 10 and 100 Kbytes/sec.

Figure 2 shows the result of the experiments. The $\mathrm{X}$-axis shows the topologies and the Y-axis shows the percentage of feasible mappings. Each bar in the figure presents for each topology the percentage number of APCGs the method is able to map given a distance. As can be seen, $96 \%$ of the cases, the mapping technique is able to map the 500 APCGs with a distance of only 5 hops for all the topologies tried. It means that the path length of the header flit 1 can be reduced from the diameter of the topology to 5 hops reducing the network overhead.

${ }^{1}$ Flit stands for flow control digit and represents the unit of data that is transmitted over a logical link (channel) in a single step. 
Table 1. Best routing algorithm for a traffic type

\begin{tabular}{|l|l|}
\hline Traffic Type & Best Routing Algorithm \\
\hline \hline Random Traffic & XY \\
\hline Hot-Spot Traffic & Odd-Even \\
\hline East-Dominated Traffic & West First \\
\hline West-Dominated Traffic & East First \\
\hline Transpose Traffic & Negative First \\
\hline
\end{tabular}

\section{Efficient Path Computation for Source Routing}

After the cores have been mapped on the NoC platform which supports distance constrained source routing, the next step will be to compute efficient paths for all the communicating pairs of cores. For each source core these paths will be stored as a table in the corresponding resource (core) to network interface (RNI). The RNI will use this table to append the path in the header flit of the packet. Beside avoiding deadlocks, the computed paths should also avoid congestion and uniformly distribute traffic among the links in the network as much as possible.

\subsection{Routing Algorithm Selection}

A large number of deterministic and adaptive routing algorithms are available for deadlock free routing in mesh topology NoCs. The most famous among these are XY, Odd-Even, West First and North Last routing algorithms. XY is a deterministic routing algorithm and allows a single path between every pair of nodes. The other algorithms are partially adaptive routing algorithms and prohibit the packets to take certain turns, but allow path adaptivity for most pairs. It has been shown that no single routing algorithm provides best performance for all types of traffic. Table 1 gives relatively best routing algorithm for some specific types of communication traffic [6].

A traffic is called West-Dominated if majority of communication (considering number of communications and communication volume) is from east to west. We analyze and classify the traffic using the mapped APCG and select the most appropriate routing algorithm. The analysis uses the relative position of source and destination cores and the communication volume between pairs [6].

\subsection{Path Computation}

One can easily compute a path for each communication pair using the routing algorithm selected using the analysis in the previous subsection. In the case of deterministic routing the only path available is selected. In the case of partially adaptive routing algorithm the path is constructed by making a choice with a uniform probability at all intermediate routers where a choice among multiple admissible ports is available. Our study has shown that communication traffic 


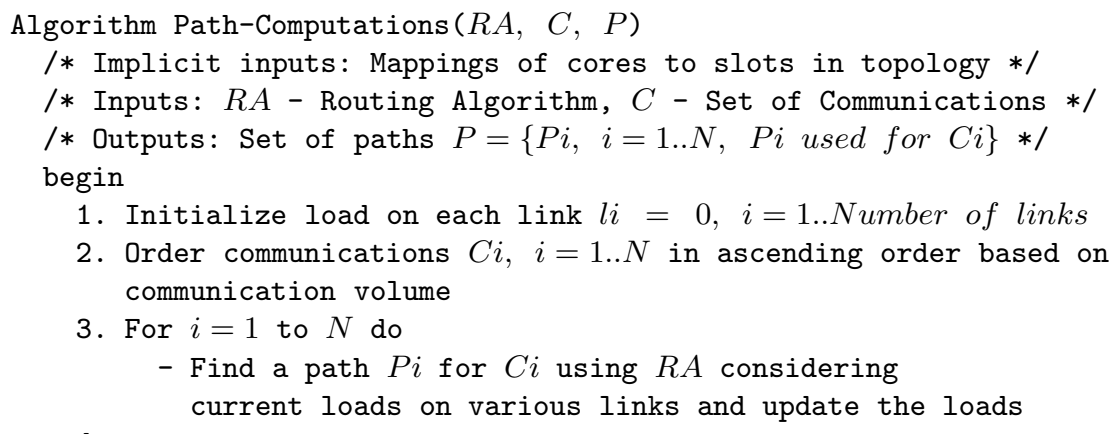

Fig. 3. Pseudocode of the algorithm for path computation

type is rarely pure. For example, it is rare to have an application with pure WestDominated traffic. To handle this we use adaptivity of the routing algorithm to balance load on the links and avoid/reduce congestion. Figure 3 describes a constructive algorithm to achieve this.

All the communications are sorted in ascending order according to their communication volume. Then in each iteration a path is computed for one communication using the selected routing algorithm. At every router where there exists a choice among multiple output ports, the port is selected if a lower load is already mapped to the corresponding output link. We keep updating the estimated load on links after each iteration. It has been shown that this methodology leads to efficient paths for communication [6].

\section{Evaluation and Results}

For evaluation purposes, we have evaluated the proposed approach using a set of random traffic scenarios. Each traffic scenario has been generated as described in section 3.1. For each scenario we have computed a random mapping, a nearoptimal unconstrained mapping and a near-optimal constrained mapping for 5 hops. The Figure 4 shows the performance results in terms of latency and throughput for two of such traffic scenarios. In this figure the random mapping, the near-optimal unconstrained mapping and the near-optimal distance constrained mapping have been labeled as RNDMAP, UNCONSTDISTMAP and MINDISTMAP respectively.

The performance evaluation has been carried out using a NoC simulator developed in SDL language [6. The simulator implements a NoC Platform based on a 2-D $7 x 7$ mesh topology and source routing. The simulated NoC also uses wormhole switching with a packet size fixed to 10 flits, the input buffers have capacity for keep 4 flits. We have used the source packet injection rate (pir) as load parameter. A Matlab based tool has been developed to compute efficient paths for source routing as described in Section 4. For each load value, latency and throughput values are averaged over 20,000 packets drained after a warm-up of 2000 packets drained. 


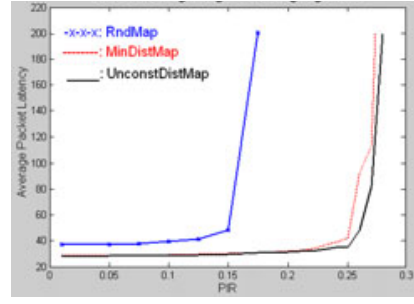

a) Latency

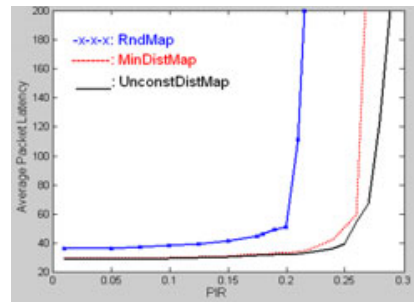

c) Latency

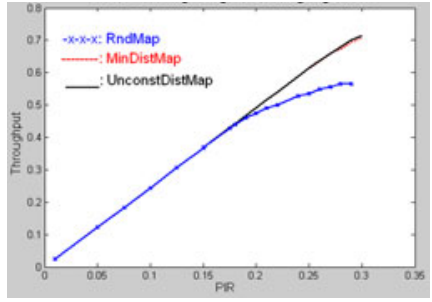

b) Throughput

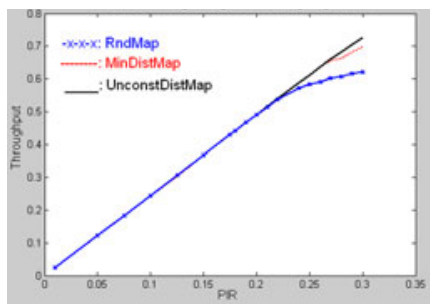

d) Throughput

Fig. 4. Simulation results for two random APCGs

Figure 4(a) and Figure 4(c) show the simulation results for average latency in cycles. These figures show that at both low traffic loads and high traffic loads both the UNCONSTDISTMAP and MINDISTMAP present similar behaviour and save more than $20 \%$ cycles and $25 \%$ over RNDMAP respectively.

The throughput results, measured in packets/cycle, are shown in Figure 4(b) and Figure 4(d). As we can look at the throughput achieved by the MINDISTMAP is almost the same as the throughput achieved by the UNCONSTDISTMAP and much higher than the throughput achieved by a RNDMAP close to saturation.

Therefore, the evaluation results show that is possible reduce the path length of the header flit at least to half of the network diameter without significantly degradation of the performance.

\section{Conclusions and Future Work}

We have addressed the application mapping problem for NoCs when the communication infrastructure is pre-designed as 2-D mesh topology using source routing and the path length header field is delimited up by a number of hops significantly less than the network diameter. In such a scenario we have demonstrated that our distance constrained mapping technique is able to map $96 \%$ of applications tried with a distance constraint less than half of the network diameter. We have proposed an efficient method, based on existing distributed deadlock free routing algorithms, to compute efficient paths required for source routing. The simulation based evaluation results show that our distance constrained mapping gives more than $20 \%$ latency improvement over random mapping at low traffic loads. The saturation points traffic load also shows around $25 \%$. Performance 
degradation as compared to path length constraint is negligible at low communication traffic and saturation packet injection rate is also only reduced by just $5 \%$.

To the best of our knowledge this is the first attempt to consider core mapping for NoC platforms based on source routing. As future work, we plan to use intelligent heuristic search method to further lower the path length thus reducing bandwidth underutilization of NoC. We are also working on methods to improve computed paths for better link load balancing. Testing this approach on large realistic applications will also demonstrate the feasibility of our approach.

\section{References}

1. Benini, L., De Micheli, G.: Networks on chips: a new soc paradigm. Computer 35(1), 70-78 (2002)

2. Chang, J.M., Pedram, M.: Codex-dp: co-design of communicating systems using dynamic programming. In: Proc. Design Automation and Test in Europe Conference and Exhibition 1999, March 9-12, pp. 568-573 (1999)

3. Murali, S., De Micheli, G.: Bandwidth-constrained mapping of cores onto noc architectures. In: Proc. Design, Automation and Test in Europe Conference and Exhibition, February 16-20, vol. 2, pp. 896-901 (2004)

4. Tornero, R., Orduña, J.M., Palesi, M., Duato, J.: A communication-aware topological mapping technique for nocs. In: Luque, E., Margalef, T., Benítez, D. (eds.) Euro-Par 2008. LNCS, vol. 5168, pp. 910-919. Springer, Heidelberg (2008)

5. Mubeen, S., Kumar, S.: On source routing for mesh topology network on chip. In: SSoCC'09: 9th Swedish System on Chip Conference (May 2009)

6. Mubeen, S.: Evaluation of source routing for mesh topology network on chip platforms. Master's thesis, School of Engineering, Jönköping University (2009)

7. Palesi, M., Holsmark, R., Kumar, S., Catania, V.: Application specific routing algorithms for networks on chip. IEEE Transactions on Parallel and Distributed Systems 20(3), 316-330 (2009)

8. Hu, J., Marculescu, R.: Energy-aware mapping for tile-based noc architectures under performance constraints. In: ASPDAC: Proceedings of conference on Asia South Pacific Design Automation, pp. 233-239. ACM, New York (2003)

9. Goossens, K., Radulescu, A., Hansson, A.: A unified approach to constrained mapping and routing on network-on-chip architectures. In: Third IEEE/ACM/IFIP International Conference on Hardware/Software Codesign and System Synthesis, CODES+ISSS '05, September 2005, pp. 75-80 (2005)

10. Tornero, R., Sterrantino, V., Palesi, M., Orduña, J.M.: A multi-objective strategy for concurrent mapping and routing in network on chip. In: Proc. of the 23rd IEEE International Parallel and Distributed Processing Symposium, May 25-29 (2009)

11. Flich, J., López, P., Malumbres, M.P., Duato, J.: Improving the performance of regular networks with source routing. In: Proceedings of the 2000 International Conference on Parallel Processing, p. 353. IEEE Computer Society, Los Alamitos (2000)

12. Minton, S., Johnston, M.D., Philips, A.B., Laird, P.: Solving large-scale constraintsatisfaction and scheduling problems using a heuristic repair method. In: AAAI, pp. 17-24 (1990) 